\title{
MERITS OF LEŚNIEWSKI TYPE NOMINALISM
}

\author{
CONTENTS
}

1. Two traditions of nominalism

2. Some merits of an Leśniewski type nominalism

3. Merits, problems and solutions 
For the sake of explaining the merits of a Leśniewski type nominalism, it should be made clear what is meant by „nominalism” and what the characteristics of this special type of nominalism are. To the first question we can find quite a lot of mutually inconsistent answers. Therefore I will just explain the distinction between two different nominalistic traditions which I hold to be fundamental. I think we should not just focus on the question which so-called abstract entities are rejected but as well look for basic entities nominalists rely on.

\section{Two traditions of nominalism}

\subsection{The Aristotelian nominalist tradition - the primacy of first substance}

Primarily, nominalism was a position of medieval philosophers who claimed for a primacy of the Aristotelian first substances, single nameable things. The radical nominalism of Roscellin is said to hold the other categories just to be a flatus vocis, the mere sound of the voice. More important was the moderate nominalism. Its most important advocate William Ockham ${ }^{1}$ held some categories (primary and secondary substances and sensible qualities) to have res absolutae as correlates, all others only to be mental qualities, signs of a mental language. So universals are in many cases just signs of a mental language. The paradigm for res absolutae are first substances. Typical for this doctrine is an analysis of simple sentences into three parts: subject, copula and predicate. The example 'Sortes est homo' can be formalized in Leśniewski's system „Ontology” simply as 'Sch'. 'Sortes' names someone we all hope to know and "homo" names all of us and many other people including Socrates as well.

Medieval Aristotelianism was present in very different versions during the centuries, at least as an opponent to be criticized. The characterized versions of Aristotelian nominalism reappear in a fully formalized version for the first time in Leśniewski's system called „Ontology”. Actually Leśniewski worked under a double influence of Aristotelian thinking: mediated by Brentano and Łukasiewicz. But the leading question of his logical work was quite different.

\footnotetext{
${ }^{1}$ For Ockham's ontology: [11].
} 
Under the influence of Łukasiewicz, Leśniewski started to scrutinize Russell's Paradox. Leśniewski was convinced that the paradox stems from a wrong definition of "class" and a lack of precision. Therefore he tried to find an absolutely precise logical language and the „correct" definition of „class". The correct concept of a class according to Leśniewski is the common sense conception of wholes or heaps. Instead of a class of trees we just have the word 'tree' as a name for all single trees. If we want something more, a class, we do not need another category but Leśniewski just gives us all trees as a whole. For this heap of trees we can use a single name 'the class of trees' instead of the general name 'tree'. A set of trees just would be a heap of trees that need not contain all the trees. An element is a part of the heap. Then every class and every set is its own element and we can avoid Russell's paradox. About the absolutely exact inscriptional logical language little can be said in the context of such a general paper because it is very complicated. As a foundation of his class theory, „Mereology”, Leśniewski developed an advanced propositional calculus, „Protothetics”. As a calculus of names he defined the so-called "Ontology" that is similar to Ockham's theory of supposition. Very roughly it is a substitute for a predicate calculus.

In his early works Leśniewski tried to develop „Mereology” in an ordinary language version. His dissatisfaction with this led him to the construction of the other systems.

To give a very short characterization of Leśniewski's systems some comments on "Ontology" will be made.

In „Ontology” we have names and sentences as basic categories. On this basis we can define functor categories. The best entrenched basic functor for the system „Ontology" has already been used. It is ' $\varepsilon$ '. The functor ' $\varepsilon$ ' combines two names to form a sentence. If we take again ' $s \varepsilon h$ ' this sentence is true if and only if ' $s$ ' stands for a single name denoting something and ' $h$ ' stands for a single or general name denoting the same thing - possibly amongst others.

One old, quite long but suggestive axiom of "Ontology" is the axiom $\mathbf{O}$ in Leśniewski's essay „Über die Grundlagen der Ontologie” (p. 114; in [14], p. 609):

$$
\Pi w a(w \varepsilon a \equiv \Sigma b(b \varepsilon w) \wedge \Pi b c(b \varepsilon w \wedge c \varepsilon w \supset b \varepsilon c) \wedge \Pi b(b \varepsilon w \supset b \varepsilon a)) .
$$

Based on Leśniewski's ideas, Kotarbiński built up a radical nominalism, called „Reism”, „Concretism” or „Pansomatism”. Kotarbiński tried to get along even without states of affairs and without any moments. Ardently he condemned universals and classes. Words that seem to stand for such entities 
are onomatoids and sentences that contain them have to be replaced by such sentences composed of a copula and names of things. Kotarbinski might be called the modern Roscellin.

As a quite recent version of a moderate Aristotelian nominalism Kripke's causal theory of reference in Naming and Necessity can be interpreted. Like Leśniewski and Ockham, he emphasized the role of singular names in language. By doing so he recapitulated the primacy of first substance. His attempt to interpret terms for natural kinds and sensible qualities the same way is similar to Ockham's acceptance of secondary substances and sensible qualities. Although Kripke accepts sets, his affinity to substitutional quantification is a further similarity to Leśniewski whose interpretation of his special quantifiers was as well guided by the use of names.

So much for the Leśniewski type nominalism. For all the mentioned ontologies in the Aristotelian tradition Leśniewski's systems can be used as a basis for a formalization.

\subsection{The phenomenalistic nominalism}

The other main stream of nominalism is a phenomenalistic one. Locke may be counted as a forerunner, Berkeley as its founder and Hume as Berkeley's follower. Primary substances are displaced by bundles of their qualities. Russell later gave this tendency a formalization, when he let definite descriptions do the job of singular names.

In Berkeley's subjective perspective „esse est percipi”. A very similar perspective can be found in Goodman's phenomenalistic systems „A Study of Qualities", his dissertational thesis, and later the more famous The Structure of Appearance. Different to Berkeley's perspective are Goodman's exclusions of subjects and Berkeley's God. Of course Goodman had a more sophisticated solution for Berkeley's problem to avoid abstraction. In his system The Structure of Appearance Goodman constructed all entities out of so-called qualia. Qualia are minimal qualitative parts of the stream of experience of a single subject ([7], p. 95 and 135).

But does this suspiciousness of abstraction suffice to call this position „nominalistic"? Indeed there are stronger reasons: Goodman took over the Leśniewskian programme to avoid the use of distributive classes as a fundament of mathematics. And Goodman even clinged to Leśniewski's method of using collective classes, these heaps or wholes, instead of distributive classes. Goodman didn't call them classes but sums of individuals. The first step to this nominalistic programme was a famous essay of Goodman and 
Quine called „Steps toward a Constructive Nominalism”. The further main steps were The Structure of Appearance, and the essay „A World of Individuals". During his intellectual development Goodman changed his criteria for nominalism several times. At first, in his thesis "A Study of Qualities" he called Carnap's Position in Der logische Aufbau, with its not repeatable Elementarerlebnissen or "erlebs”, nominalistic and his system, with its repeatable qualia, realistic. In his later system that avoided classes (the old one used classes, but just classes of individuals), he suggested calling such systems nominalistic. Then, systems that use classes without a possibility of reformulation should be called platonistic. The most prominent development of Goodman's point of view is the work of Rolf A. Eberle.

To sum up the historical remarks, nominalism is a family resemblance concept. But the works of the most famous nominalists resemble Leśniewski's systems in very different ways. If we do not want to split nominalism in two different traditions or in a modern and two ancient traditions we have to rely on Leśniewski.

After this sketch of the development of two different nominalistic traditions we can come to the merits of the Leśniewski type nominalism. The merits of Goodman's tradition that should of course not be neglected, are better known.

\section{Some merits of an Leśniewski type nominalism}

\subsection{A help for orientation}

One special advantage of centering on Leśniewski should already be evident. It helps us to get a survey of nominalistic positions. Depending on Quine's and Goodman's criteria of ontological commitment one could not even understand the key-notes of the Aristotelian branch of nominalism. The missing link between the medieval nominalistic tradition and authors like Goodman is the work of Leśniewski. When we miss the central role of Leśniewski, we are very likely to end with some ad hoc criteria for nominalism with very strange results of application.

I think there is no use in calling positions „nominalism” when there is no sufficient correspondence to the name giving medieval nominalism. Goodman has on several occasions conceded that there is no non-arbitrary correspondence between his setting of problems and the medieval ones. For instance, Goodman wrote in The Structure of Appearance on page 137: „It might be argued that the rejection of classes naturally carries with it the 
rejection of qualities because both classes and qualities are abstract. [...] If the argument is based rather on the analogy between the relationship of a class to its many members and that of a quality to its many instances, an equally good analogy can be drawn between the relationship of a class to its many members and that of a concrete individual to its many qualities."

In my view, Leśniewski's system „Mereology” is the link between Goodman and Leśniewski and Leśniewski's system „Ontology” is another link between Leśniewski and Ockham. In this way going via Leśniewski, we can produce a form of network between the main so-called nominalists.

To resume it in a way paradoxically: Leśniewski expresses the essence of nominalism.

\subsection{The Primacy of Development}

A second point is the primacy of Leśniewski's work. Goodman, informed by Quine, Tarski and Carnap, was influenced by Leśniewski, at least in his development of his "Calculus of Individuals" and his inscriptional syntax.

After these more or less historical reasonings, let us now come to the heart of the problem. The Leśniewski type nominalism and the phenomenalistic one share some central problems, but nevertheless both deal with problems of their own. So we have to check, which one of the solutions to the common problems are better and which of the particular problems seem to be relevant or real problems. And for the relevant problems, again, we have to ask which solutions can suffice.

\section{Merits, problems and solutions}

The shared problems are problems how to reduce all commitment of formal sciences like mathematics or the formal framework of theoretical physics.

The particular problems of both accounts have a longer tradition. They are independent of scientific developments because they are tied to ordinary language. For the Aristotelian tradition, there is the question of a fundamentum in re of predication. If we were asked why we say that Socrates is a man, could we really just answer that Socrates is amongst the things named by the word 'man'?

Another problem would be to find a substitute for the so-called states of affairs.

For authors of the phenomenalistic tradition there are totally different problems. They have to fill the gap between subjective judgements of an ex- 
periencing single subject and objective judgements about the external world. Furthermore they want to avoid abstraction and end up with a problem of concretion.

\subsection{Nominalistic foundations of science}

Let us start with the technical problems that are difficult but are more or less common amongst the modern advocates of the different traditions.

If we want to have a foundation of Mathematics and want to get along without sets and classes we can, roughly speaking, choose between two strategies. In his essay „Why I am not a Nominalist” ([2], see p. 96ff) John P. Burgess spoke of a revolutionary and an hermeneutic nominalism. The revolutionary one tells us to build up mathematics anew and thereby give up all these mathematical theories for which we cannot find a nominalistic foundation. Hermeneutic nominalism leaves mathematics as it is, but tries to interpret it in a nominalistic way. According to Karl Marx the second strategy, i.e. . just to interpret but avoid all changes, is quite typical for philosophers. Both nominalistic traditions never earnestly tried to build up mathematics anew, like for instance Paul Lorenzen once tried.

Nevertheless Leśniewski was a hermeneutic nominalist with a revolutionary formal language. In hermeneutic nominalism, as it is defined by Burgess, there is something more than a nominalistic interpretation of mathematics: it is the claim for a deep structure in mathematics that is acceptable for a nominalist. It is exactly this which Leśniewski claims for his collective classes. He thinks that the sum of all things denoted by a name is the correct definition of class and the sum of some things denoted by a name is the correct definition of set. In Leśniewski's „Mereology”, the class-membership-relation is replaced by the part-whole-relation. Leśniewski tried to give evidence for his thesis that the leading intuition of other logicians in their introduction of classes was that of collecting different things to form a whole. He tried to prove this by checking the commentaries these logicians gave when introducing classes. So, according to Leśniewski, this whole could be nothing but all the things taken together as their sum.

Goodman on the contrary has never claimed that the sums of individuals of his calculus are classes. In fact, in the early version he used classes of individuals to define them ([9]). But later on he tried to replace the use of set-theoretic formulae by his calculus of individuals. He was looking for a reformulation of set theory but not for a hidden nominalistic deep structure as is lined out by Burgess. Nevertheless Goodman's nominalism can be rather called hermeneutic than revolutionary. 
So the common aim of Leśniewski and Goodman is not to deliver a new mathematics, but to give a reformulation of mathematics that is more sensitive to the question of whether classes should be introduced than the normal accounts are.

If we just have a calculus of individuals or Leśniewski's „Mereology” and try to replace the classical set-theoretical foundation of mathematics we will run into trouble quite soon. If we manage to find a substitute for the natural numbers that does not require second order logic the next severe problem is to reach the real numbers. Normally we need the axiom of choice, an axiom of power set or an axiom of replacement.

I don't know how the work done by Goodman could in any way suffice to produce something like Edmund Landau's „Grundlagen der Analysis” on the sole basis of the calculus of individuals.

Leśniewski has something more to offer as a foundation than Goodman has. With his "Mereology" he can do as much as Goodman. But since he was aware of the fact that this will not suffice, Leśniewski has tried to strengthen his system "Ontology" to a so-called non elementary "Ontology". This form of "Ontology" uses variables for functors. Because of the German invasion of Poland there has not been saved enough of Leśniewski's writings to be sure how far he worked out this programme. We have to rely on lecture notes and the impressions reported by his friends and students.

In part two, chapter four of the book S. Leśniewski's Lecture Notes we can find the notes of Bolesław Sobociński from the term 1928/1929. There Leśniewski tried to develop a substitute for Peano-axiomatization of natural numbers and tried to develop the system further. ${ }^{2}$ Later on, John Thomas Canty has defined an axiom of infinity and used it to define the natural numbers ([3]). J. G. Kowalski ([12]) and C. C. Davis ([4]), two students of Sobociński, worked on versions of the Axiom of choice as supplements for non elementary ontology. Kowalski proved it to be equivalent to Zorn's lemma and the Well ordering theorem. ${ }^{3}$

It would be a progress for the work on Leśniewski's main topic, the foundation of mathematics, if there were once written a form of summary of the results and an investigation about the strength of the available methods. Up to now it is difficult to find out how far we cant get with it.

In one respect Goodman's comprehensive system The Structure of Appearance fits better with modern mathematics. Goodman reconsiders on

\footnotetext{
${ }^{2}$ Słupecki had a second try on this topic in his essay [22], p. 71.

3 In the last case he proved actually a so-called „rule equivalence”. For the implication of the Well ordering theorem by the Zorn's lemma he had to repeat his presupposition for higher functors.
} 
several occasions the possibility to define isomorphisms that enable him to switch from one type of basis to another one. This setting reminds on the focussing on structure that is quite usual in modern mathematics. The primacy of names and naming in Leśniewski's system is in contrast to this mathematical tendency to center on structure.

Although Goodman's calculus of individuals seems to supply a weaker foundation of mathematics than Leśniewski's systems, Goodman's system is in a much higher degree than Leśniewski's, influenced by mathematical structures. Besides the already mentioned isomorphisms of bases we should think for instance of the topological elements in his theory of qualia and the calculus of simplicity.

A very important but not nominalistic recent development in mereology is David Lewis' book Parts of Classes. Although it is not nominalistic and uses distributive classes it founds its mereological sums not on classes but on named pluralities of things - just like Leśniewski. Lewis argues for the value of an independent mereology in the following way: „So I claim that mereology is legitimate, unproblematic, fully and precisely understood. All suspicions against it are mistaken. But I claim more still. Mereology is ontological innocent." ([18], p. 81)

\subsection{The special problems of Goodman's phenomenalistic system}

The next point to discuss is the particular problems of the two different traditions.

Goodman wanted to define a constructional system in the tradition of Carnap's system „Der logische Aufbau der Welt” that has a phenomenalistic basis. In opposition to Carnap, Goodman wanted to have a „realistic” basis that consisted of repeatable qualitative entities, so-called qualia. Goodman's setting is very similar to Berkeley's. But Goodman is less dogmatic than Berkeley. In fact he does his best to avoid any dogmatism at all. Nevertheless, let us first imagine a philosopher, we may call him Badman, who has the same position as Goodman but is a dogmatist like Berkeley. Now we can ask our leading question ,is the problem posed by the philosopher a relevant one?" to Badman and his system. I think the construction of the world of science and the worlds of art or social communities out of the stream of consciousness of one single subject by means of first order logic, one basic relation (,alied with"), and the calculus of individuals, could seem to be an idle and superfluous investigation. 
But Goodman is not Badman. He never tried to use one single system to solve all problems of the whole world. Goodman never wanted to build up THE constructional system of THE world, but several systems to solve limited, definite problems. In his later work he even spoke of „Ways of Worldmaking” and preferred to call his system „Structures of Appearance”. Secondly by considering his isomorphisms Goodman always tried to be able to switch to other bases like physicalistic or particularistic ones. Although this sounds fairly liberal there is still his dependency on the form of constructional systems. And there is another problem still remaining. The question is whether an ontology that is built on such a multiplicity of perspectives is still an ontology or in the end just a mathematical structure. And in fact in the end Goodman has very little to offer besides a simple basic relation without any intuitive foundation and a calculus of individuals. In his later works Goodman tried to refrain from this tendency of focussing on a formal perspective.

\subsection{Leśniewski's foundations: Lebenswelt and ordinary language}

Prima facie Leśniewski was even more limited in his interests to logical questions than Goodman. But his logic is naturally rooted in the Aristotelian tradition and its primacy of names. Leśniewski always insisted that his basic functor ' $\varepsilon$ ' should be read as the Polish 'jest' or the Latin 'est'. Of course, Leśniewski was aware of the problems that result from using his system "Ontology" as a formalization of natural language. If we do not limit the contexts strictly, then we easily end up with problems like this: We call both Warszwa in 1830 and that of today 'Warszwa'. But they are not the same. In a logical calculus this leads to contradiction. But if we forget for a moment these problems of limitation of contexts Leśniewski's system "Ontology" in its simple forms seems to function as an abbreviation of the way the copula 'is' and names are used in some natural languages.

Leśniewski once wondered if he should apply his system only to phases of things to avoid problems. I would prefer to limit contexts, because I think this is, what we do in ordinary language. For instance, I do not name a phase of a person, but the person himself or herself. And I know how to limit contexts, in order to avoid contradictions.

The reliability of the contexts of Lebenswelt ensures Leśniewski that his axioms in "Mereology" and in "Ontology” are not arbitrary ones like Russell's. In fact he believed in them as true sentences like Aristotle did in his first principles of Analytica Posteriora. 


\subsection{A fundamentum in re for predication}

Leśniewski's system „ontology” delivers a formal framework both for moderate Aristotelian nominalism like Ockham's and for Kotarbiński's radical nominalism, called reism. It seems to be an open question, which ontology he himself would have favoured. His system "Ontology" supplies just the basic categories of names and sentences. But he nevertheless was not satisfied with Kotarbiński's reductionism and with its tendency to materialism. When we use Leśniewski's system „Ontology” without any supplements as a formalization of natural language we have to explain why the use of names alone suffices to explain predication. Is there for instance nothing more to say about, why roses are red, than that roses are amongst the things named 'red'? By the way, a set-theoretical extensionalism does not have to offer much more.

The moderate Aristotelian nominalism has to supplement Leśniewski's system „Ontology" with categories for individual accidents or even for moments or nowadays so-called tropes in general. Already Kazimierz Ajdukiewicz ([1]) and later Czesław Lejewski ([16]) suggested bicategorial systems.

Peter Simons ([20]) has developed different versions of a Leśniewski type calculus of names with new categories added. First he introduced individual accidents and then the so-called truth-makers that in his ontology replace the better entrenched states of affairs ([17]). The difficulties in these settings lie in the modal aspects of some conceptions that tend to break the limits of extensionalism. And Leśniewski was a convinced extensionalist. Furthermore Simons' truth-makers tend to increase in number. When we accept a few we will end up with quite a lot. Needless to say most nominalists are not exactly thrilled by this idea.

One merit of a moderate Leśniewski type nominalism could be that it offers the old question about the fundamentum in re of predication in a modernized version and initiates a research programme. And another nominalistic merit is that it supplies natural bounds for the development of more and more moments in Ockham's and Kripke's agreement about the Aristotelian categories that should be supported. A merit of an acceptable theory of truth-makers would be to get rid of states of affairs.

I think these problems are quite natural ones for any ordinary language philosophy. To work with different categories seems to fit closer with a common sense conception of existence then to reduce violently everything to a single mode of existence. 


\subsection{New solutions for old dilemmas}

It seems quite natural to ask how a Leśniewski type nominalism deals with problems that have been paradigmatically solved by Russell, Quine and Goodman.

One old dilemma of nominalism, the inability to formulate criticism without using the taboo-words of realistic or so-called platonistic positions have been solved by Russell and Quine in a well-known way. Singular definite descriptions replace names. Ontological commitment is tied to bound variables. "A theory is committed to those and only those entities to which the bound variables of the theory must be capable of referring in order that the affirmations made in the theory be true", as Quine formulated in his famous essay „On what there is" ([19], p. 13). With these formal instruments we can deny the existence of classes without using names for them and thereby in a way accepting such entities. Goodman relied on this strategy.

As Leśniewski has a special form of quantification which is free from ontological commitments, one has to find another solution for this problem.

The second dilemma is closely linked with the first one. How can a very restricted nominalist language suffice for the discussion of ontological problems? Surely a platonist would not accept the limits of Goodman's system in order to discuss which system is to be preferred. In his late ontological essay „A World of Individuals" Goodman solves this problem by interpreting nominalistic systems as contained in platonistic ones that are in need of classes as referents of bound variables. For Leśniewski, this solution is not acceptable because he insisted that he cannot even understand what is meant by a distributive class. Furthermore Leśniewski's logic is an idiosyncrasy and is in fact not part of platonistic systems.

This situation seems quite hopeless for Leśniewski: he has to face two difficult problems that are already solved by the rival tradition of Quine and Goodman. It seems that all he can do is to reach the same level.

But in fact his situation is not that bad because the dilemmas are old ones. The solution Quine and Goodman have to offer only works if we can reconstruct an ontology in Quine's canonical notation or in the constructional systems of Carnap or Goodman. This should not be taken for granted for instance in the case of ontologies in the Aristotelian tradition.

I think it is possible to find a new solution for these problems on the basis of Leśniewski's „Ontology”. In order to do this we have to make use of the possibility of introducing new basic categories. I have already mentioned that Leśniewski's quantification is free of any ontological commitment. Leśniewski quantifies over names and sentences and possibly over higher functors or 
members of new introduced categories. But for instance even the names he quantifies over can be empty or have multiple denotation. If we derive a quantified formula - in fact there are no free variables in Leśniewski's systems - its truth may not depend on whether a name-variable denotes something or not. ${ }^{4}$

The solution for our problems could be to introduce categories for alleged entities that realists or platonists want to talk of, and to try to find translations for their sentences into a system restricted to names or terms for individual accidents. The nominalist can use these categories purely syntactically and be sure that they contain empty, not refering terms. Maybe he even can persuade his opponent by pragmatical reasoning that such categories are superfluous. These discussions do not take place in the area of formal logic but in that of ordinary language philosophy.

\section{References}

[1] Kazimierz Ajdukiewicz, „On the Problem of Universals”, [in:] Kazimierz Ajdukiewicz, The scientific World-Perspective and other Essays 1931-1963, Jerzy Giedymin (ed.), Dordrecht, Boston 1978.

[2] John P. Burgess, „Why I am not a Nominalist”, NDJFL, Vol. 24, No. 1, 1983, p. 93-105.

[3] John T. Canty, „Ontology: Leśniewski's Logical Language”, [in:] Leśniewski's Systems, Jan T. J. Srzednicki, V. Frederick Rickey (eds.), The Hague, Boston, Lancaster 1984, p. 149-164.

[4] Charles C. Davis, „An Investigation concerning the Hilbert-Sierpiński Logical Form of the Axiom of Choice", NDJFL Vol. XVI, No. 2, 1975, p. 145-183.

[5] Rolf A. Eberle, Nominalistic Systems, Dordrecht 1970.

[6] Nelson Goodman, „A Study of Qualities”, PHD thesis, Harvard 1940, published in N. Y. 1990.

[7] Nelson Goodman, The Structure of Appearance, Cambridge Mass. 1951, 3. revised edition, Dordrecht 1977.

[8] Nelson Goodman, „A World of Individuals”, [in:] N. Goodman, Problems and Projects, Indianapolis NY, 1972.

[9] N. Goodman, Henry S. Leonard, „The Calculus of Individuals an its Uses”, Journal of Symbolic Logic, Vol. 5, No. 2, 1940, p. 45-55.

[10] N. Goodman, W. V. O. Quine, „Steps toward a Constructive Nominalism”, Journal of Symbolic Logic, Vol. 12, 1947, p. 105-122.

\footnotetext{
${ }^{4}$ For Leśniewski's quantifiers: Guido Küng [13] and Peter Simons [21].
} 
[11] Matthias Kaufmann, Sätze, Dinge, Referenz und Wahrheit bei Wilhelm von Ockham, Leiden, N. Y., Cologne 1993.

[12] James George Kowalski, „Leśniewski's Ontology extended with the Axiom of Choice", NDJFL Vol. XVIII, No. 2, 1977, p. 1-78.

[13] Guido Küng, „The Meaning of the Quantifiers in the Logic of Leśniewski”, Studia Logica No. 26, 1977, p. 309-322.

[14] Stanisław Leśniewski, Stanisław Leśniewski - Collected Works, Stanisław J. Surma, Jan T. Srzednicki, D. I. Barnett (eds.), Dordrecht, Boston 1992.

[15] Stanisław Leśniewski, Lecture Notes in Logic, Jan T. J. Srzednicki, Zbigniew Stachniak (eds.), Dordrecht, Boston, London 1988.

[16] Czesław Lejewski, „A System of Logic for Bicategorial Ontology”, Journal of Symbolic Logic, Vol. 3, 1974, p. 265-283.

[17] Kevin Mulligan, Peter Simons, Barry Smith, „Truth-Makers”, Philosophy and Phenomenological Research, Vol. XLIV, No.3, 1984, p. 287-321.

[18] David Lewis, Parts of Classes, Cambridge (Mass.) 1991.

[19] W. V. Quine, „On what there is”, [in:] From a Logical Point of View, Cambridge (Mass.), 2. ed.

[20] Peter Simons, „A Leśniewskian Language for the Nominalistic Theory of Substance and Accident", Topoi 2, 1983, p. 99-109.

[21] Peter Simons, „A Semantics for Ontology”, Dialectica Vol. 39, Fasc. 3, 1985, Biel, p. 193-216.

[22] Jerzy Słupecki, „S. Leśniewski’s Calculus of Names”, Studia Logica 3, 1955, p. 7-76.

Henning Hintze

Institut für Philosophie

Universität Erlaugen-Nürnberg

Bismarckstr. 1

D-91054 Erlangen, GERMANY 